Archives de sciences sociales des religions

125 | janvier - mars 2004

Autorités religieuses en islam

\title{
Autorités religieuses en islam
}

Marc Gaborieau et Malika Zeghal

\section{(2) OpenEdition}

Journals

Édition électronique

URL : http://journals.openedition.org/assr/2883

DOI : $10.4000 /$ assr.2883

ISSN : 1777-5825

Éditeur

Éditions de l'EHESS

Édition imprimée

Date de publication : 1 janvier 2004

Pagination : 5-21

ISBN : 2-222-96741-4

ISSN : 0335-5985

Référence électronique

Marc Gaborieau et Malika Zeghal, « Autorités religieuses en islam », Archives de sciences sociales des religions [En ligne], 125 I janvier - mars 2004, mis en ligne le 05 décembre 2005, consulté le 01 mai 2019. URL : http://journals.openedition.org/assr/2883 ; DOI : 10.4000/assr.2883 
Arch. de Sc. soc. des Rel., 2004, 125, (janvier-mars 2004) 5-21

Marc GABORIEAU

Malika ZEGHAL

\section{AUTORITÉS RELIGIEUSES EN ISLAM}

Depuis la fin des années soixante-dix, la montée de l'islamisme a mis au premier plan le poids de la religion dans la politique. Pour gérer les diasporas musulmanes de plus en plus nombreuses qu'ils abritent, les gouvernements des pays occidentaux ont dû, d'autre part, chercher parmi elles des interlocuteurs, en particulier des autorités religieuses qui leur paraissaient insaisissables. Tout cela a relancé la réflexion sur la nature et les formes de l'autorité religieuse en islam. Nous disons bien " relancé », car ce n'est pas d'aujourd'hui que les historiens et les islamologues se sont interrogés sur ces questions. L'imbrication de la religion et de la politique en islam est un thème que certains orientalistes comme Bernard Lewis - bien avant les spécialistes de l'islamisme - affectionnaient particulièrement (Sadowsky, 1997). Plus généralement les médiévistes s’interrogent depuis longtemps sur les institutions religieuses de l'islam et la façon dont l'autorité s'y exerce (Gardet, 2002). Le présent numéro, prenant du champ par rapport aux controverses récentes, voudrait donc renouer avec des réflexions plus anciennes et présenter une vue dans la longue durée de l'autorité religieuse en islam et des personnes ou des institutions en lesquelles elle s'incarne.

\section{Les trois pôles de l'autorité religieuse en islam}

Précisons d'abord ce que nous entendons par autorité religieuse en islam.

L'autorité au sens abstrait - distinguée du pouvoir brut, de la force et de la violence - s'entend du droit d'imposer l'obéissance au nom de valeurs en principe partagées par ceux qui lui sont soumis (Hardy, 1986, pp. 42-43). Mais les contributeurs de ce numéro ont aussi pris ce terme dans d'autres sens. Il y a d'abord un second usage abstrait, illustré ici par Baudoin Dupret, pour désigner la force exécutoire d'un texte de référence ou d'une décision de justice. De ces sens abstraits dérive un sens concret qui a été choisi par la plupart des contributeurs : les personnes et les institutions qui incarnent cette autorité abstraite et la mettent en œuvre. Nous avons placé au premier plan cette acceptation en mettant « autorités 
religieuses » au pluriel dans le titre de ce recueil. Nous renouons ainsi, dans une autre perspective, avec un autre numéro plus ancien de la présente revue (Archives de sciences sociales des religions, 1983).

Dans l'islam, la notion même d'autorité est problématique, et cela pour deux raisons. L'une est théologique : elle ne peut se définir que dans une «constante référence à Dieu, seul souverain véritable, qui communique aux hommes non l'essence de son autorité et de son pouvoir, mais son Commandement » (Gardet, 2002, p. 281) ; comment peut-on alors comprendre que des hommes et des institutions possèdent une autorité ? En second lieu en dépit de ces prétentions totalisantes de la théologie, beaucoup de sphères et de sources d'autorité - nous y reviendrons à la fin de cette introduction - relèvent d'un autre domaine que celui de la religion, se rattachant aux coutumes ethniques en matière de hiérarchie sociale et de structures familiales, ou à des modèles politiques qui ne relèvent pas de l'islam ; toute autorité n'est pas ipso facto religieuse. Nous nous limiterons donc ici à celles qui se définissent en termes religieux, ou qui ont un impact sur l'organisation de la pratique religieuse.

Il n'y a pas en islam de définition claire et univoque d'une sphère spirituelle distincte d'une sphère temporelle (Gardet, 2002, p. 280). En matière de religion, il n'existe pas, dans la doctrine, d'institution unique comme une Église ayant le monopole du domaine religieux, ni de classe d'individus consacrés dédiés intégralement aux fonctions religieuses; on ne peut, comme dans le cas iranien par exemple, parler que métaphoriquement d'un "clergé » (Richard, 1983). On ne saurait trouver un interlocuteur unique à qui s'adresser : si la référence à Allah est la seule à fonder l'autorité, la volonté d'Allah suit au moins trois lignes de délégation - qui ne sont pas sur le même plan - pour s'exercer dans la société humaine.

Les deux premières mettent en concurrence deux voies d'accès à la connaissance de la volonté divine, qui peuvent être cumulées par la même personne comme nous allons le voir : dans la terminologie traditionnelle elles sont appelées exotérique (zâhirî) et ésotérique (bâtinî).

L'exotérique vient toujours au premier plan : c'est la voie médiate de l'interprétation de la volonté divine parvenue aux hommes à travers le Coran et les traditions prophétiques (hadîth). Elle s'appuie sur les sciences religieuses ('ulûm, sg ' ilm) dont la langue technique est l'arabe et qui sont enseignées dans des établissements spécialisés appelés madrasa (pl. madâris). La science reine est le droit (fiqh), qui s'appuie sur ces sciences auxiliaires que sont " la grammaire, l'exégèse coranique et la science des traditions »; le juriste (faqîh) est le personnage central de l'islam; la théologie a une importance secondaire, à la différence de ce qui se passe dans la tradition chrétienne (Grandin, Gaborieau, 1997, Introduction). Ceux qui maîtrisent ces sciences sont appelés oulémas ('ulamâ, sg. 'âlim) ou docteurs de la Loi. Ils sont traditionnellement considérés comme les successeurs du Prophète et les dépositaires de l'interprétation autorisée de la volonté divine. Ils ont une triple fonction : ils transmettent le savoir, encadrent le culte et sont les spécialistes du droit qui font fonctionner la justice. Dans ce dernier rôle, ils peuvent être des experts (muftî) qui rendent des avis juridiques ou fatâwâ ( $\operatorname{sg}$ fatwâ), ou de juges ou cadis (qâdî) qui siègent dans les tribunaux.

La voie ésotérique est fondée sur un accès immédiat au divin par l'expérience mystique qui, outre l'extase qui unit à Dieu, procure une sorte de révélation par les rêves et surtout par ces sortes d'illuminations - complétant la révélation 
prophétique - que sont le dévoilement (kashf) et l'inspiration (ilhâm). Ceux qui atteignent ces expériences, et qui sont aujourd'hui généralement regroupés dans des ordres mystiques ou confréries, sont appelés soufis (sûfî) (Popovic, Veinstein, 1996 [cf. Arch. 100.128]). Alors que l'arabe est la langue technique des sciences exotériques, le soufisme utilise très largement, dans ses textes doctrinaux et surtout dans sa poésie, les grandes langues véhiculaires que sont le persan et le turc ainsi que les vernaculaires des différentes régions du monde musulman. Tout au long du Moyen-Âge comme pendant la période moderne, le saint soufi est resté une autorité charismatique indispensable aux souverains pour conquérir et légitimer leur pouvoir (Berque, 1958 et 1982 ; Digby, 1986 ; Touati, 1994).

La coexistence de ces deux premières voies est complexe. Elles peuvent être réunies dans le même homme, comme c'était généralement le cas depuis le haut Moyen-Âge et jusqu'à l'orée du XIX ${ }^{\mathrm{e}}$ siècle. Les mêmes personnes jouissaient alors d'une double autorité : leur science de l'interprétation des textes était confortée par le charisme qu'ils tiraient de leur expérience mystique; on les a appelés les " oulémas/soufis » (Gaborieau, 1989). Mais cette alliance, que l'on rencontre souvent encore aujourd'hui, n'est pas toujours présente. Elle est optionnelle, car on peut choisir d'être seulement docteur de la Loi ou seulement soufi. Dans l'histoire, le soufisme a pris corps et a été reconnu par l'orthodoxie après la constitution des sciences religieuses (Gril, 2000). Depuis le XVIII ${ }^{\mathrm{e}}$ siècle avec la naissance du wahhabisme et depuis l'émergence du modernisme au XIX ${ }^{\mathrm{e}}$ siècle - deux frères ennemis souvent en collusion comme on l'a vu au début du $\mathrm{XX}^{\mathrm{e}}$ siècle dans le salafisme de Rashîd Ridâ (Mervin, 2000, pp. 170-172) - le soufisme a été dévalorisé comme une innovation blâmable (bid'a), entachée de superstition ; il a même fait l'objet d'interdiction en Arabie saoudite et dans la Turquie post-ottomane. Ainsi, la coexistence des voies exotérique et ésotérique peut être conflictuelle, surtout dans le cas du sunnisme, la tradition majoritaire dans l'islam (1); dans le chiisme, la dimension ésotérique a largement pénétré les sciences religieuses et le culte, laissant peu de place à un conflit entre les deux voies.

Ainsi ces deux pôles potentiellement conflictuels - divisés entre eux, et scindés à l'intérieur d'eux-mêmes entre ces courants rivaux que sont les écoles juridiques et théologiques d'un côté, les multiples ordres mystiques de l'autre - ne sont-ils pas subsumés sous une ombrelle unique qui poserait une instance spirituelle opposée à une instance temporelle, cette dernière étant le pouvoir politique.

En fait, le pouvoir politique constitue un troisième pôle religieux. Le souverain est l'ombre d'Allâh sur la terre ; il a hérité de son Commandement; il est de sa responsabilité d'assurer le bon fonctionnement des institutions religieuses, en particulier la mise en application de la Loi par les cadis qu'il a nommés, comme le souligne par exemple en Inde l'historiographie moghole (Hardy, 1986, pp. 43-45). Il a une autorité religieuse qui lui permet de réprimer les excès et les hérésies, et d'arbitrer les conflits des deux autres pôles religieux, comme nous le verrons plus bas dans le cas de l'empire ottoman et des États qui lui ont succédé. En ce sens le pouvoir politique revendique l'autorité religieuse suprême, les oulémas et les soufis lui étant juridiquement subordonnés, et dépendant souvent de lui pour leur financement (Gaborieau 1989). Mais, en un autre sens, le pouvoir est dépendant d'eux

(1) Pour une présentation générale des grands courants de l'islam comme le sunnisme et le chiisme et leurs divers courants, voir MERvin, 2000. 
pour sa légitimation et sa protection spirituelle, car les religieux, comme on le verra notamment dans le cas du chiisme duodécimain, ont beaucoup de ressources pour déjouer ses tentatives de mainmise. On peut même parler d'un partenariat entre pouvoir politique et oulémas, qui peut devenir inégal, favorisant l'un ou l'autre de ces partenaires, ou allant jusqu'à la rupture de ces fragiles alliances, selon les contrainte politiques du moment (Keddie, 1972).

Entre les trois pôles de l'autorité religieuse il n'y a donc pas de hiérarchie univoque fixée une fois pour toute, mais une dialectique incessante qui joue sur plusieurs points de vue et brouille les rôles sans qu'il y ait réellement de vainqueur. Nous y reviendrons longuement dans la suite de cette introduction.

\section{Présentation du numéro}

C'est cette dialectique que le présent recueil se propose d'étudier dans une perspective historique longue. Comment l'autorité religieuse est-elle définie et appropriée ? Est-elle contrôlée institutionnellement, peut-elle être aussi remise en question, réformée, détruite, ou reconstruite ? Comment peut-elle émerger dans la sphère sociale autour de figures charismatiques, devenir politique ou s'abstenir d'intervenir politiquement? Peut-elle être plurielle, changer de mains, se partager? Comment cette autorité est-elle perçue, discutée, acceptée, contestée, suivie?

Le recueil commence, dans une première partie, par une réflexion générale sur les trois grands pôles de l'autorité religieuse en islam et la façon dont ils s'articulent au cours de l'histoire. Un premier article pose la question de la détention de l'autorité religieuse dans les débuts de l'islam, en décrivant le processus de transfert de l'autorité religieuse des premiers califes à celui des lettrés spécialistes du savoir religieux, qui se constituèrent progressivement comme corps de statut au cours des trois premiers siècles de l'islam (Christian Décobert). Le texte suivant analyse la façon dont se hiérarchisent les corps des oulémas et des soufis, et comment ils s'articulent au pouvoir politique dans le contexte sunnite ottoman; c'est la seule formation politique médiévale qui ait essayé de construire une hiérarchie religieuse ordonnée, la 'ilmiyye, qui se révélera cependant instable (Nathalie Clayer). Puis vient une étude sur la spécificité du chiisme duodécimain arabe et iranien par rapport au sunnisme : elle décrit l'émergence au XIX ${ }^{\mathrm{e}}$ siècle d'une autorité religieuse supérieure, celle du marja', qui parvient à déjouer toutes les tentatives de mainmise politique (Sabrina Mervin). Revenant au contexte sunnite, un dernier article montre comment l'autorité des mystiques définie par la tradition médiévale est toujours vivante dans l'Égypte contemporaine (Rachida Chih).

La deuxième partie rassemble des études de cas. Les deux premières sont situées en contexte musulman. Arrive en premier lieu un article sur la sécularisation de la Turquie après 1920 et l'ambiguïté de ses effets, puisque l'appartenance à l'islam a été gardée comme un critère indispensable de la turcité, et que l'État a établi un contrôle serré de la vie religieuse (Hamit Bozarslan). Puis vient une analyse de la production des fatâwâ en Indonésie et des rapports des divers 
courants des oulémas indonésiens aux grands centres d'autorité religieuse dans le monde musulman (Niko Kaptein). Les trois textes suivants s'attardent sur trois exemples de construction d'autorités religieuses en diaspora en France (Franck Frégosi), aux États-Unis (Aminah Mohammad-Arif) et en Grande-Bretagne (article de Philip Lewis introduit par une note d'Aminah Mohammad-Arif).

En épilogue vient une analyse nuancée des différentes facettes de l'autorité religieuse telles qu'elles se manifestent dans le contexte des tribunaux égyptiens (Baudoin Dupret).

\section{Le poids des autorités politiques}

Ce qui frappe d'abord à la lecture de ces articles, c'est le poids de la politique en matière de religion, même aujourd'hui. On aurait pu croire qu'il s'agissait d'un trait médiéval effacé par la modernité après la disparition des empires ottoman et moghol. Il n'en est rien, même dans le cas d'un État proclamé laïque comme la Turquie et dans le cadre des pays chrétiens d'Occident qui accueillent des diasporas musulmanes.

Pour reprendre la question dans une perspective historique, un des paradoxes de l'autorité religieuse en islam réside dans l'existence de professionnels religieux qui forment un corps d'oulémas officiels, dans la plupart des pays musulmans, comme le soulignent en particulier les articles de Nathalie Clayer et de Hamit Bozarslan. Les débats des historiens sur la constitution des élites des fuqahâ', spécialistes du droit, et des muhaddithûn, spécialistes de la Tradition (hadîth), aux débuts de l'islam, sont encore contradictoires. Les recherches de Muhammad Qasim Zaman sur les débuts de la période abasside (750-1258) remettent en cause l'hypothèse d'Ira Lapidus selon laquelle autorité religieuse et politique étaient séparées et en compétition (Lapidus, 1975 ; Crone \& Hinds, 1986 ; Zaman, 1997 ; Dakhliya, 1998 ). Il y a semble-t-il, lorsque l'on s'arrête sur les liens entre autorité religieuse et autorité politique, une tension constante entre la multiplication des sources d'autorité religieuse et leur unification autour d'un pôle exclusif. Ainsi, en revenant à la période formative de l'islam, Christian Décobert nous rappelle, dans sa contribution, que l'autorité religieuse fut d'abord investie par les califes qui reproduisaient en ce sens le modèle prophétique. Contrairement à l'acception commune, et en convergence avec les analyses de Patricia Crone, il donne un exemple parlant de cette confusion de l'autorité politique et religieuse dans l'usage de l'expression Khalîfat Allâh dès l'accession au pouvoir du premier calife « bien guidé » et sous les Omeyyades. Le décalage - qu'on peut interpréter comme une division du travail - entre autorité politique et religieuse émerge et s'accentue sous les Abassides, sans que le califat se défasse cependant de ses attributs religieux. La tension entre ces deux fonctions et ces deux types de légitimité peut ainsi mener au conflit - comme dans l'épisode de la mihna (2) - mais n'est pas toujours contradictoire.

(2) Ce terme, signifiant littéralement "épreuve", désigne une persécution d'une vingtaine d'années infligée à partir de 833 par le calife Ma'mûn, partisan de la doctrine théologique mu`tazilite, aux oulémas qui refusaient d'y adhérer (MERvin, 2000, pp. 92-93). 
Christian Décobert souligne l'importance des paramètres historiques et des contraintes sociales pour expliquer le conflit entre ces deux fonctions et la mise en place d'une institution religieuse comme cadre formel qui, à cette étape de l'histoire de l'islam, se définit comme un ensemble de " chaînes concurrentes de virtuoses» qui construisent des «mémoires religieuses autorisées ». L'exemple plus proche de l'empire ottoman permet de comprendre la mise en place d'une véritable hiérarchie religieuse officialisée par l'État, une série de chaînes d'autorités fortes, mais jamais extensives. Nathalie Clayer nous explique ainsi que le système hiérarchisé des oulémas passés par l'institution de la 'ilmiyye - telle qu'elle s'est mise en place au $\mathrm{XVI}^{\mathrm{e}}$ siècle - ne couvrait pas l'ensemble de ce qui constituait l'autorité religieuse, même si le souverain ottoman tirait en partie sa légitimité de la loi sacrée, dont les oulémas officiels étaient les gardiens et les interprètes.

Cet exemple est essentiel parce qu'il est au fondement de l'organisation de l'institution religieuse dans plusieurs des nations qui sont issues du démembrement de l'empire ottoman. C'est vrai non seulement pour l'organisation du groupe des oulémas durant les périodes pré-tanzimât, mais aussi pour les périodes postcoloniales où la réforme des institutions religieuses a été poussée à bout par les États indépendants. L'exemple des réformes sécularisatrices d'Atatürk, dont Hamit Bozarslan décrit toute l'ambiguïté, se décline plus tard en des versions différentes dans des pays comme l'Égypte ou la Tunisie (Zeghal, 1999a et 1999b); l'on sait que les États postcoloniaux se sont inspirés - positivement ou contradictoirement du précédent turc qui a constitué une véritable référence. Ainsi dans l’Égypte nassérienne, la réforme d'al-Azhar par le régime des officiers libres a mis en place le contrôle étroit des oulémas par le pouvoir, contrôle qui s'est accompagné d'une division du travail entre autorité religieuse et autorité politique, la première étant soumise à la seconde (Zeghal, 1996). Le régime des officiers libres avait besoin de l'autorité religieuse azharie, car il était incapable de s'imposer sur le plan religieux. Il faisait autorité sur le plan profane à travers le charisme de Nasser et l'idéologie nationaliste, mais aussi par l'usage de la violence d'État qui s'est exercée entre autres sur les oulémas à qui il donnait le rôle de détenteurs exclusifs de l'autorité religieuse. À partir des années 1970, l'émergence de l'islamisme, produit d'une dérégulation de la sphère religieuse par l'État, s'exprime à travers l'émergence d'autorités religieuses nouvelles et multiples, qui ne sont plus nécessairement sous tutelle de l'État. Mais en prenant au sérieux la concurrence des islamistes de tous bords, les cheikhs d'al-Azhar ont transformé leur institution et lui ont redonné poids et autorité à partir des années 1980 .

On retrouve des phénomènes analogues plus loin à l'Est au Pakistan ou en Indonésie. Dans le premier pays, au cours des années soixante-dix et quatre-vingt, Bhutto puis le général Zia cherchèrent dans l'islam une légitimation de leur pouvoir. Ils furent alors amenés à intervenir de plus en plus dans le domaine religieux : sous Bhutto le Parlement pakistanais a défini en 1974 qui était musulman et qui ne l'était pas en déclarant la secte des Ahmadiyya non musulmane; concrétisant les promesses de Bhutto, Zia a islamisé (au moins superficiellement) le droit, levant de force l'aumône légale et étendant le contrôle étatique sur l'enseignement religieux des madrasa et le fonctionnement des sanctuaires soufis (Gaborieau, 2000). En Indonésie, l'islam n'est pas la religion officielle, mais l'État intervient largement dans le domaine religieux. Ainsi le polythéisme est interdit et seules sont autorisées les religions monothéistes : ce qui oblige les hindous et les bouddhistes à 
se redéfinir comme monothéistes, et les animistes à se ranger sous la bannière de l'une de ces deux dernières religions s'ils refusent la conversion à l'islam ou au christianisme. En ce qui concerne l'islam, l'État prend à sa charge l'enseignement religieux et contrôle l'application du droit islamique (Feillard, 2001, pp. 165-175). Comme le montre l'article de Nico Kaptein, l'État indonésien a aussi créé en 1975 une organisation officielle des oulémas, le Majlis Ulama Indonesia.

Le rôle de l'État - non musulman en l'occurrence - est loin d'être absent en diaspora. Les exemples de l'islam en France, en Grande-Bretagne et aux États-Unis montrent la difficulté de la mise en place d'institutions musulmanes représentant une autorité religieuse, en particulier lorsque l'État la sollicite comme chez nous. La construction du CFCM - né au forceps, comme le souligne Franck Frégosi part de ce malentendu qui marque cette institution du sceau de l'incertitude quant à sa légitimité. Il peut être pertinent à ce propos de relever le contraste entre les cas de la France et des États-Unis, qui sont très différents, mais dont on voit bien les convergences dans les formes que prend l'autorité religieuse pour les musulmans qui y résident. Aux États-Unis, l'État ne sollicite que peu, et jamais explicitement, la formation d'autorités religieuses, alors que les pouvoirs publics français ont travaillé à la matérialiser durant des années (Frégosi, 1998, pp. 101-167). Dès lors, la compétition pour l'appropriation de l'autorité religieuse se manifeste sur des modes différents qui partagent cependant des points communs. Aux États-Unis, comme l'explicite la contribution d'Aminah Mohammed, elle procède d'un double travail de reconnaissance, qui s'exprime de la part de la clientèle musulmane qui reconnaît l'autorité religieuse de certains individus, ou d'organisations, mais s'exprime aussi dans la reconnaissance, au sens d'acceptation, de ces sources d'autorité par le pouvoir politique. Ainsi l'État peut-il prendre acte de l'existence d'institutions religieuses musulmanes, à des moments précis, comme il le fit à partir des années 1990, ou dans les cérémonies collectives de deuil après les attentats du 11 septembre 2001. En France, en revanche, l'État sollicite la naissance et l'unification de l'autorité religieuse, et marque même sa préférence pour certaines de celles qui ont émergé de l'initiative des croyants. Du coup sa reconnaissance par les musulmans eux-mêmes est moins affirmée peut-être, puisque l'État est directement actif: il recherche l'interlocuteur et travaille à le créer, à lui donner une identité institutionnelle. On parle souvent de la difficulté de l'islam français à se doter de représentants en lui cherchant des causes internes à la communauté religieuse : la diversité des courants théologiques ou idéologiques, celle des origines nationales, ou encore les liens forts entre certaines communautés et les États d'origine. Mais ces faits sont aussi présents ailleurs, comme aux États-Unis ou en Grande-Bretagne. Ce qui rend pénible la constitution d'autorités "reconnues" en France, c'est justement le fait qu'elles doivent être reconnues par l'État lui-même ; c'est la mise en branle du mécanisme d'émergence de l'autorité par la volonté étatique. Mais la différence entre les "modèles" américain et français n'est pas toujours si nette. Aux États-Unis, depuis le 11 septembre 2001, l'État intervient dans la reconnaissance et la non-reconnaissance de certaines des sources de l'autorité religieuse musulmane, par la surveillance, la mise en suspicion de certaines organisations religieuses et l'encouragement d'autres. 


\section{Vitalité des instances d'interprétation}

Face à ces empiètements de l'État, les oulémas qui ont en charge l'interprétation de la volonté divine ont eu recours à des stratégies inventives d'adaptation et d'organisation.

En islam, les textes du Coran et de la Sunna ou tradition prophétique sont ceux qui font autorité de manière primordiale et reviennent à l'autorité suprême et unique, celle d'un Dieu qui ne peut souffrir d'associés. En un sens, le tawhîd, affirmation de l'unicité divine, instaure une distance infinie entre Dieu et les hommes ; il fait de l'autorité religieuse, si on pousse le principe à ses limites, une capacité exclusivement divine, qui ne peut appartenir aux hommes. L'idée selon laquelle le pouvoir "ne revient qu'à Dieu", ou qui dicte que l'obéissance ne peut se faire qu'envers celui qui obéit à Dieu, réside au fondement de cette utopie ; il faut bien pourtant introduire la médiation humaine dans l'expression même de l'exclusivité de l'autorité divine, d'autant que le tawhid implique aussi l'égalité d'accès à la parole divine, telle qu'elle se dégage des textes révélés, pour tous les croyants.

Ces interprètes des textes divergent sur la manière de les lire et de résoudre les questions qu'ils posent. Ainsi les traditions dites "faibles" font évidemment moins autorité que les autres, mais certains contextes peuvent les rendre plus fortes pour certains (Brown, 1996). Le mécanisme de l'abrogation (naskh) des versets permet aussi de recomposer l'autorité du texte révélé, même si, considéré comme un tout, il est celui qui fait le plus autorité. Il est ainsi possible de redonner voix et autorité potentielle à l'interprète qui fait médiation entre le texte et sa réception. Le texte sacré ne fait jamais autorité "seul", mais à travers sa lecture, son insertion dans la prière, le sermon, la leçon, l'essai historique, la harangue politique et d'autres espaces et véhicules d'intervention (Gaffney, 1994). Au-delà des textes sacrés, les sources et les manifestations de l'autorité religieuse sont multiples et s'entrecroisent: les normes juridiques, inscrites dans les quatre grandes écoles de droit (Mervin, 2000, pp. 64-81), ou relevées directement dans le texte révélé et la tradition; les fatâwâ, anciennes ou nouvelles, que certains oulémas qualifient aujourd'hui d'"avis" pour ne pas en surévaluer l'autorité.

Même si le travail d'interprétation est à la base individuel, les oulémas ont dû adopter des stratégies collectives. Dans la confrontation directe avec l'État d'abord. $\mathrm{S}$. Mervin montre qu'en chiisme comme en sunnisme, les oulémas ont eu et ont encore un rôle de contestation des pouvoirs en place. Elle permet de comparer deux modèles : la centralisation étatique avec l'empire ottoman a réduit le pouvoir de contestation des oulémas sunnites, en les privant d'une partie de leur indépendance. C'est ce qui explique que rares sont les oulémas contestataires qui appartiennent à l'institution religieuse officielle dans le monde sunnite aujourd'hui. La contribution de S. Mervin met en valeur le contraste avec le chiisme duodécimain, pour lequel le processus inverse s'est produit: dans l'histoire de cette secte, l'autorité religieuse, qui était représentée dans un premier temps par l'imam descendant du Prophète, se désincarne ensuite avec la disparition du $12^{\mathrm{e}}$ imam. Elle fut donc reconstruite progressivement, aboutissant au $\mathrm{XIX}^{\mathrm{e}}$ siècle à l'émergence du personnage du marja ; plus récemment, poussant à bout cette construction de l'autorité religieuse, on vit apparaître avec Khomeiny le wilâyat $i$-faqîh, qui institutionnalise, à partir de cette autorité religieuse même, une autorité politique. On ne retrouve rien de 
semblable dans le monde sunnite aujourd'hui, où l'on imagine mal un État dirigé par les oulémas : l'islamisation du Pakistan a été faite paradoxalement par des militaires formés à l'anglaise. La seule exception est l'exemple éphémère des Talibans en Afghanistan qui ont mis sur pied un État dirigé par les oulémas ; encore cette construction reposait-elle sur le charisme d'un illuminé, le Mollah Omar, qui était en fait très largement téléguidé par les généraux des services de renseignements pakistanais; et elle ne fut possible que parce que les madrasa subventionnées par l'État pakistanais avaient mis sur le marché une masse pléthorique de chômeurs intellectuels (Dorronsoro, 2000 ; Roy, 2000).

Mais généralement la confrontation à l'État n'est pas aussi directe. Les oulémas s'organisent en associations pour former des sortes de lobbies face à la fois au pouvoir politique, et à la société qu'ils veulent réislamiser. C'est le cas, en Égypte, d'abord depuis qu'ils se sont libérés en partie du carcan imposé par Nasser. On ne peut qu'être frappé par le prestige actuel d'al-Azhar dans le monde musulman. Pourquoi cette centralité ? Plusieurs facteurs entrent en compte, qu'on ne détaillera pas ici, mais nous voudrions revenir sur un aspect particulier, qui permet de réfléchir sur les effets de la "globalisation" (Fandy, 1999). De nombreux débats, qui prennent la forme de guerres de fatâwâ entre oulémas azharis, trouvent des échos au-delà même d'al-Azhar depuis les années 1990 (Zeghal, 1997). Certains oulémas azharis ont aussi transcendé l'institution; ils se sont positionnés géographiquement et institutionnellement à l'extérieur d'al-Azhar et de l'Égypte et ont atteint une notoriété globale. On peut penser plus particulièrement à Youssef Qaradhawi, qui offre ses avis sur la chaîne de télévision al-Jazira mais aussi sur internet. Sur son site internet personnel, on peut lire sa longue autobiographie, où les racines azharies et égyptiennes sont mises en avant de manière très visible. Avant lui, feu cheikh Kichk, lui aussi formé à al-Azhar, se faisait entendre sur cassette audio dans l'ensemble du monde arabe. Le prestige que beaucoup de musulmans dans le monde reconnaissent à Qaradhawi renforce celui d'al-Azhar et inversement. Le succès du passage de cette figure individuelle à la notoriété internationale s'ancre en grande partie dans l'autorité et le prestige de son institution d'origine, tout comme dans la distance qu'il a su prendre avec celle-ci. Force centrifuge, Al-Azhar est aussi un espace de réception des futurs oulémas étrangers. D'autres oulémas célèbres occupent d'autres niches symboliques de la tradition de transmission du savoir religieux. Hamza Yusif, un américain converti à l'islam, a ainsi fondé son institut, qui, situé en Californie, s'apparente à la structure de la madrasa. Cet institut, nommé Zaytûna, fait ainsi référence à la traditionnelle institution de transmission du savoir religieux en Ifriqiyya (actuelle Tunisie). L'occupation de l'espace religieux, retraditionnalisé, au niveau global, la mise en valeur d'une histoire longue, expliquent ainsi le rayonnement renouvelé de certaines institutions religieuses; ce qui a permis, en retour, d'offrir un nouveau lustre à certains oulémas sur le plan global. La centralité de grands lieux historiques de production et de transmission du savoir religieux montre qu'il est possible de nuancer les hypothèses selon lesquelles l'autorité religieuse musulmane serait aujourd'hui fragmentée et décentrée, en particulier à travers le phénomène de la production d'autorités religieuses sur internet (Roy, 2002). La persistance des autorités religieuses officielles et leur capacité d'adaptation suggèrent alors que l'on a probablement surévalué le phénomène de l'auto-proclamation par les autorités religieuses nouvelles que représentent les islamistes, et négligé des phénomènes importants de réinstitutionnalisation de l'autorité religieuse. Ainsi, en Égypte, 
certains prêcheurs ont dû, après avoir fait des études modernes et techniques, passer par les facultés religieuses d'al-Azhar pour se donner une légitimité et un statut religieux, que leur qualité de prêcheur ne suffisait pas à leur conférer. Autre exemple : le ministre français de l'intérieur, Nicolas Sarkozy, a rendu visite le 30 décembre 2003 au Grand Imam d'al-Azhar, le cheikh Tantawi, pour obtenir une fatwâ qui dispense les femmes musulmanes fréquentant les écoles publiques françaises de porter le voile - par ailleurs considéré par le Grand Imam comme « obligation divine »; cette démarche du ministre était motivée par le fait que le CFCM, dominé en partie par l'Union des Organisations Islamiques de France, ne pouvait lui donner le feu vert. Les rapports journalistiques de la rencontre sont intéressants en eux-mêmes : ils présentent al-Azhar comme le «Vatican de l'islam sunnite », et le cheikh Tantawî comme la "principale autorité de l'islam sunnite » (3), attribuant à al-Azhar - comme le fait la rencontre Sarkozy-Tantawi elle-même - une autorité suprême en matière religieuse. Cette prétendue suprématie est pourtant continuellement remise en question par l'existence d'un champ religieux aujourd'hui éminemment pluriel - et par là même concurrentiel - en Égypte même (où la fatwâ de Tantawi est contestée par nombre d'oulémas d'al-Azhar) comme au niveau global. Après une longue période de déclin, qui prend ses racines dans la modernisation et l'occidentalisation des savoirs dans le monde musulman, on peut ainsi noter un phénomène de réinstitutionnalisation des espaces anciens de transmission du savoir religieux et de légitimation religieuse.

Ce va et vient entre les grands centres historiques du monde arabe et les régions plus excentrées est particulièrement bien illustré par le cas de l'Indonésie. Ainsi, Niko Kaptein, décrivant les centres de production des fatâwâ pour l'Indonésie aux $\mathrm{XIX}^{\mathrm{e}}$ et $\mathrm{XX}^{\mathrm{e}}$ siècles : la Mecque d'abord, puis le Caire - dans la sphère d'al-Azhar ou hors d'elle - où l'on peut trouver des autorités modernistes, et enfin de plus en plus en Indonésie même. Là, les fatâwâ peuvent émaner d'individus ou d'associations. Ces dernières peuvent être d'inspiration gouvernementale (comme la Majlis Ulama Indonesia déjà mentionnée) ou volontaires ; ce dernier cas illustre le phénomène de réorganisation des communautés musulmanes colonisées depuis la fin du $\mathrm{XIX}^{\mathrm{e}}$ siècle. L'Indonésie possède deux associations volontaires d'oulémas qui ont une forte influence sur la société et sur la politique et incarnent deux sensibilités différentes. La plus puissante est la traditionnaliste Nahdlatul Ulama (NU) qui laisse une grande place au soufisme et aux traditions indonésiennes, mais est aussi devenue un parti politique illustré par l'ex-président Abdur Rachman Wahid (Feillard, 1995). La seconde est la moderniste Muhammadiyyah qui s'est très largement inspirée des salafites égyptien comme Rashid Rida; elle est par conséquent hostile au soufisme et prend pour inspirateurs les leaders modernes du ProcheOrient (Feillard, 2001, p. 164).

Le sous-continent indien représente un autre cas de figure. Comme le monde turco-iranien en général - et à la différence de l'Indonésie - il est moins tourné vers la Mecque et exploite davantage ses propres ressources qui se rattachent aux traditions iranienne et centre-asiatique. Après la répression de la révolte des Cipayes en 1857-1858, les oulémas indiens se sont réorganisés pour défendre la communauté musulmane face à l'occidentalisation et à la résurgence de l'hindouisme. L'exemple a été donné par l'école de Deoband fondée en 1867 (Hardy, 1972; Metcalf, 1982;

(3) Voir par exemple Libération du Mercredi 31 décembre 2003, p. 8. 
Zaman, 2002). Elle illustre un courant réformiste indigène : elle a minimalisé le soufisme qui a été épuré du culte des saints, conservé son attachement à l'école de droit hanafite, et a été à la pointe du combat politique contre les Anglais, dominant l'Association des oulémas de l'Inde qui depuis sa fondation en 1919 fait figure de représentant officiel de la communauté musulmane. Le bureau central des fatâwâ de Deoband et celui de l'association des oulémas, dont les décisions sont régulièrement publiées, font figure de cour de justice alternative pour les musulmans pieux qui refusent les tribunaux d'État. Le code de conduite deobandi est très largement suivi ; il a notamment été adopté par l'organisation du Tablîgh qui propage les idées deobandies dans le monde entier (Gaborieau, 1997) ; d'une façon plus générale les Deobandis ont été très influents dans l'émigration, comme le montre en particulier l'article de Philip Lewis. La branche pakistanaise du mouvement est aussi extrêmement influente : elle est devenue un parti politique dès 1956 ; elle comporte une aile radicale minoritaire qui a inspiré les Talibans. Le courant deobandi est donc une vaste nébuleuse où l'on trouve aussi bien des quiétistes comme les Tablîghî que des radicaux qui prêchent la guerre sainte. Les autres écoles (voir encore Metcalf, 1982), qui ont aussi leurs madrasa et leur bureau de fatâwâ au Pakistan comme en Inde, se sont définies en réaction à Deoband, qu'elles soient salafites comme celle des Ahl-i hadith, ou traditionalistes comme les Barelwis qui insistent particulièrement sur la dimension soufie comme nous allons le voir bientôt (Sanyal, 1996), et qui sont aussi devenues des partis politiques au Pakistan. Mais il nous faut auparavant mentionner Maududi (1903-1979) sans qui ce tableau de l'islam indo-pakistanais ne serait pas complet. Autant que l'Égyptien Hassan al-Banna dont il est le contemporain, il peut être considéré comme l'ancêtre de l'islamisme (Carré, 1984). Choisissant en 1947 d'émigrer au Pakistan, il a voué le reste de sa vie à tenter de faire de ce pays l'État islamique dont il fut historiquement le premier théoricien; il fut le grand catalyseur des débats sur l'islamisation du Pakistan; son organisation politico-religieuse, la Jamâ'at-i islâmî, devint en 1956 un parti politique qui servit de modèles aux partis deobandi et barelwi (Nasr, 1996). La Jamâ'at-i islâmî a eu un succès limité au Pakistan. Elle est par contre importante dans l'émigration où elle a établi un réseau transnational influent notamment dans les milieux éduqués (Grare, 2001): elle fut en Angleterre à l'origine de l'affaire Rushdie; les travaux d'Aminah Mohammad montrent son influence dominante et paradoxale sur les intellectuels musulmans en Amérique du Nord.

\section{La résilience du soufisme}

On aurait pu croire que le soufisme - qui, comme nous l'avons vu, fait l'objet de vives critiques de la part des modernistes, des salafites et des islamistes - allait connaître un déclin souvent annoncé (Gilsenan, 1967). Il n'en est rien ; il contribue à informer beaucoup plus qu'on ne croit la vie des gens du peuple comme des lettrés.

La structure du soufisme montre clairement comment l'autorité religieuse permet de construire la relation entre maître et disciple : elle ne passe généralement pas par l'étude médiatisée du texte, mais par la pratique du compagnonnage 
(suhbâ) qui est un véritable cadre de socialisation; Abdellah Hammoudi (1997) la met au fondement de la production et de la continuité de l'autoritarisme au Maroc. Comme nous le montre la contribution de Rachida Chih, qui donne la parole aux disciples du cheikh soufi en Égypte, l'importance des qualités individuelles, jusqu'à leur inscription dans le comportement, dans le vêtement, l'attitude, et le corps, permet d'incorporer la piété comme qualité intrinsèque, naturelle, et donc durable. La formation théologique, qui peut être marginale (une madrasa de province) ou centrale (al-Azhar par exemple), la généalogie, la transmission par le cheikh du "secret", marquent aussi le lien d'une autorité religieuse à une autre, plus ancienne, et peut renforcer l'autorité du cheikh dans un processus d'accumulation de ses sources. Dans ce cas, l'autorité religieuse se construit par la proximité du cheikh au divin et par le renforcement des liens sociaux entre ceux qui le suivent. Les confréries soufies ou ordres mystiques ont ainsi créé, dans leurs hospices et autour des tombes de leurs saints, des institutions qui sont des lieux de sociabilité importants pour la plus grande partie des musulmans (Popovic, Veinstein, 1996, pp. 205-241 ; Mayeur-Jaouen, 2002).

Le soufisme reste vivant non seulement dans ses formes populaires, mais aussi parmi les lettrés. La plupart des cheikhs d'al-Azhar sont aussi des mystiques. C'est ce qui rend cette université historique attractive pour les étudiants étrangers qui restent fidèles au soufisme traditionnel : ainsi un soufi naqshbandi de Delhi, Abû Hasan Zaid Fârûqî (1906-1993) qui fut influent en Inde, au Pakistan et en Afghanistan - sans compter ses disciples occidentaux, notamment italiens - raconte dans son autobiographie comment il choisit d'aller étudier à al-Azhar, où il trouva des membres de sa confrérie parmi ses enseignants, plutôt que de subir en Inde les critiques de Deobandis (Gaborieau, 1990, pp. 240 ; 264). Le soufisme informe aussi certaines des grandes associations d'oulémas de l'Asie méridionale dont nous avons parlé plus haut : la Nahdlatul Ulama en Indonésie, et l'école de Barelwis en Inde : le fondateur de cette dernière, Ahmad Ridâ Khân Barelwî (1856-1921) était lui-même un grand soufi et un redoutable polémiste qui défendit le soufisme traditionnel contre ses détracteurs (Sanyal, 1996).

Dans les pays d'origine, le soufisme a su se réorganiser pour se défendre et reste sans doute majoritaire. En Égypte les confréries prennent de multiples formes, y compris celle d'associations de bienfaisance (Chih, 2000). En Inde et en Indonésie, avons-nous vu, il prospère sous l'ombrelle des associations d'oulémas traditionalistes. Au Pakistan les soufis ont aussi créé des associations spécifiques qui fonctionnent en accord avec les réseaux des Barelwis dans le sous-continent (Buehler, 1998) comme à l'étranger, étendant leurs ramifications jusqu'en Turquie (Gaborieau, 1990, p. 264). Par contre en diaspora le soufisme traditionnel peine à exporter ses institutions: les articles d'Aminah Mohammad et Philip Lewis montrent que ce sont les courants réformistes comme les Deobandis, ou carrément fondamentalistes comme la Jamâ'at-i Islâmî, qui tiennent le haut du pavé ; les ordres occidentalisés (Hamès, 1996 [cf. Arch. 100.128]), qui attirent surtout un public européen ou américain (converti ou non), ne peuvent combler ce manque. 


\section{Le rôle des croyants en terre d'islam et en diaspora}

On prête beaucoup d'attention à l'autorité religieuse et à ses manifestations, mais peu au fait que parfois, elle ne fonctionne plus, ou s'émousse auprès de ceux qui avaient pu y croire. En effet, les croyants font partie de la fabrication de l'autorité religieuse. Vers qui se tournent-ils ? Et pourquoi ? On a tendance à y porter peu d'attention, mais Rachida Chih nous rappelle comment l'autorité du cheikh de la confrérie est interprétée, vécue par les disciples, jusque dans les manifestations physiques qui se cristallisent dans l'attitude corporelle du chef de la confrérie. Cette attention portée au rôle des croyants dans la fabrication de l'autorité religieuse devrait aussi pouvoir se porter ailleurs que dans l'étude de l'islam mystique des confréries, ou de ce qu'on appelle l'islam "populaire" parce qu'il rend justement visibles les émotions vives et les comportements du croyant. Mais pourquoi ne pas s'y attacher dans d'autres milieux et à propos d'autres types de comportement religieux ? L'islamologie s'est peu interrogée sur ce qui fait l'autorité d'un imam, d'un prêcheur, ou d'un chef politique islamiste pour leur clientèle. Pourtant les enquêtes ethnologiques sont prometteuses; elles montrent que les fidèles de base sont beaucoup plus actifs dans la quête d'autorité que ne voudraient le faire croire les écrits des lettrés (Gaborieau, 1993).

L'étude des diasporas récentes - dans les contributions de Franck Frégosi, Philip Lewis et Aminah Mohammad - révèlent l'existence d'une véritable quête de l'autorité religieuse, dans certains cas très laborieuse, non seulement par les croyants eux-mêmes, mais aussi par les autorités publiques, en particulier l'État comme nous l'avons vu plus haut. Ces articles montrent qu'il a fallu, pour les migrants, créer leurs autorités religieuses, les institutionnaliser dans un cadre local et national au sein duquel on assiste paradoxalement pour les religions moins récentes, en particulier le christianisme, à une désinstitutionalisation du religieux, ou du moins à un affaiblissement des grandes institutions traditionnelles qui ont perdu de leur autorité. C'est dire la difficulté de la mise en place d'institutions musulmanes, en particulier lorsque l'État la sollicite comme en France. Nous avons déjà relevé le contraste entre la France et les États-Unis pour ce qui est du rôle de l'Etat. Les mêmes remarques peuvent être faites pour la quête des fidèles pour une appropriation de l'autorité religieuse. Aux États-Unis, comme l'explicite la contribution d'Aminah Mohammad, elle procède d'abord d'une reconnaissance de la part de la clientèle musulmane qui entérine l'autorité religieuse de certains individus, ou d'organisations. En France, du fait de la sollicitation de la part de l'État, la communauté musulmane est plus passive et attend un peu trop des autorités publiques, comme il ressort des discussions qui ont eu lieu au moment de la mise en place du CFCM. L'article de Philip Lewis montre aussi l'esprit d'initiative de la diaspora indo-pakistanaise pour se créer des institutions islamiques et former sur place des cadres religieux adaptés au contexte.

Il est en revanche un point sur lequel la convergence est frappante entre l'Angleterre, la France et les États-Unis : la figure de l'imam, qui est marginale en terre d'islam, est devenue importante et multifonctionnelle en diaspora. La mosquée est aujourd'hui l'espace primordial de rencontre des musulmans et de l'émergence de l'autorité religieuse. C'est probablement une des raisons pour lesquelles le processus français de consultation, ou d'istishâra, a fait le choix de 
faire émerger les autorités religieuses à partir des mosquées, notamment en fonction de leur surface en mètres carrés. La personne de l'imam est devenue une source fondamentale d'autorité musulmane, si bien que l'on s'empresse aussi parfois de la lui retirer. Comme le souligne Franck Frégosi, aucun imam n'est représenté dans le récent CFCM. L'imam est devenu, aux États-Unis, dans le cas de la communauté musulmane indo-pakistanaise, comme le dit si bien Aminah Mohammad "représentant de la mosquée", mais la compétition autour de son pouvoir pousse les représentants "laïques" de la mosquée, à le minimiser. La mosquée se veut alors espace de présence et de représentation visible de l'islam, mais reste fragmentée en tant que lieu de l'autorité, d'autant que celle-ci est aussi représentée par les croyants qui souvent élisent, aux États-Unis, leurs représentants religieux au sein de la mosquée.

Alors qu'en France et aux États-Unis, les institutions de transmission du savoir musulman ne sont pas très développées, en Grande-Bretagne la madrasa est devenue un lieu de fabrication et un véhicule important de l'autorité religieuse. Aminah Mohammad-Arif présente d'abord brièvement la situation d'ensemble des musulmans britanniques. Puis Philip Lewis fait ensuite une étude détaillée de la formation en Angleterre même des nouveaux oulémas dans la mouvance deobandie : alors que leurs prédécesseurs restaient sans lien avec la société extérieure, ils ont dû - question de survie oblige - s'ouvrir sur le monde qui les entoure et introduire la compétence de leurs diplômés dans les institutions de savoir modernes et non religieuses pour trouver leur audience. Philip Lewis décrit pour la Grande-Bretagne une situation où la madrasa semble être devenue un lieu de fabrication et un véhicule important de l'autorité religieuse. Ces madrasa sont liées à différentes postures politiques par rapport à la société britannique. Celles qui se sont développées en Grande-Bretagne dans la mouvance deobandie sont dans un premier temps restées sans lien avec la société extérieure; mais, question de survie oblige, elles ont dû, après avoir formé des espaces enclavés, s'ouvrir sur le monde extérieur, introduire la compétence de leurs diplômés dans les institutions de savoir modernes et non religieuses pour trouver leur audience.

Ce dynamisme croissant des fidèles en diaspora pour trouver ou se créer des autorités religieuses appropriées à leur situation s'accompagne - comme le souligne en particulier l'article d'Aminah Mohammad - d'un effort personnel de réflexion et d'individuation qui se traduit en particulier dans la floraison de sites internet. Ces deux mouvements contradictoires permettent d'ouvrir le champ de l'islam en diaspora à la question de l'individuation religieuse dans ses rapports aux nouvelles formes d'institutionalisation de l'autorité religieuse (Hervieu-Léger, 2001).

\section{Les limites des autorités religieuses}

Malgré une mise en garde initiale, nous avons peut-être eu tendance à surestimer le caractère religieux des autorités qui s'imposent aux musulmans, ou qu'ils se donnent. En particulier on s'attendrait à ce que le droit, science reine de l'islam, et surtout le droit de la famille qui passe pour le dernier sanctuaire de la tradition 
islamique, soit de part en part informé par la religion. La lecture des islamistes comme Maududi nous a trop habitués à voir l'islam comme un programme détaillé couvrant tous les aspects de la vie en société.

L'article de Baudoin Dupret qui vient en épilogue de ce recueil souligne au contraire que la réalité historique est bien plus nuancée. La loi ne fonctionne pas comme une donnée homogène qui dicterait ce qu'est la réalité sociale, juridique ou politique. L'autorité du texte, de la norme, ne fait sens que dans, et en tant qu'elle est perçue, utilisée, travaillée, mise en branle dans des situations particulières. Ce que le texte de la sharî'a dit n'existe que dans sa lecture. Le texte de loi concernant le statut personnel, n'est, pour B. Dupret, pas forcément "religieux", ou ne fait pas forcément autorité parce qu'il dériverait d'une soi-disant loi sacrée. Il remet ainsi en question l'essentialisation de la loi comme sharî̀a, comme loi religieuse : il souligne que, rapportée au contexte de l'énonciation, en situation de procès par exemple, elle ne s'actualise pas comme "religieuse", comme le montrent les textes oraux transcrits lors des procès qu'il nous présente. Son analyse prouve aussi que si le contexte est primordial, le texte l'est aussi : c'est dans l'interaction entre ces deux instances que se manifeste le caractère performatif du texte, qui peut alors "faire autorité". L'article de Dupret pose ainsi une question fondamentale, au-delà même de la question de l'autorité du texte juridique et de sa contextualisation par l'accomplissement pratique de la règle. Il s'agit de savoir quelle est la place de l'autorité religieuse en général dans les sociétés musulmanes : est-elle essentielle, prépondérante, ou occasionnelle ? La question a le mérite de pousser à la réévaluation des travaux sur "l'islamisation": ils ont porté en grande partie sur les mouvements politiques et ont très vraisemblablement grossi et essentialisé le rôle de la variable religieuse. Se poser la question de savoir comment l'autorité religieuse fonctionne aujourd'hui en islam, c'est - faut-il conclure - se poser aussi la question de ses limites.

\author{
Marc GABORIEAU \\ Centre d'Études de l'Inde et de l'Asie \\ du Sud \\ CNRS-EHESS
}

\author{
Malika ZEGHAL \\ Centre d'Études Interdisciplinaires \\ des Faits Religieux \\ CNRS
}

\section{BIBLIOGRAPHIE}

Archives de sciences sociales des religions, $n^{\circ}$ 55.1, 1983, «Personnels et groupes religieux en islam », dossier coordonné par Constant HAMĖS, pp. 5-104.

BERQUE Jacques, Al-Yousi : Problèmes de la culture marocaine au XVIIe siècle, Paris, Sindbad, 1958.

BERQUE Jacques, Ulémas fondateurs, insurgés du Maghreb au XVIIe siècle, Paris, Sindbad, 1982.

BROWN Daniel, Rethinking Tradition in Modern Islamic Thought, Cambridge, Cambridge University Press, 1996.

BUEHLER Arthur, Sufi Heirs of the Prophet. The Indian Naqshbandiyya and the Rise of the Mediating Sufi Shaykh, Columbia, University of South Carolina Press, 1998. 
CARRÉ Olivier, Mystique et politique. Lecture révolutionnaire du Coran par Sayyid Qutb, Frère musulman radical, Paris, Presses de la FNSP/Éditions du Cerf, 1984.

CHIH Rachida, Le soufisme au quotidien. Confréries d'Égypte au XXe siècle, Paris, Actes-Sud, 2000.

CRONE Patricia, HINDS M., God's Caliph: Religious Authority in the First Centuries of Islam, Cambridge, Cambridge University Press, 1986.

DAKHLIA Jocelyne, Le divan des rois. Le politique et le religieux en islam, Paris, Aubier, 1988.

DIGBY Simon, « The Sufi Shaikh as a Source of Authority in Mediaeval India », in Marc GABORIEAU, éd., Islam et société en Asie du Sud, Paris, Éditions de 1'EHESS, 1986, pp. 57-77 (coll. «Purushârtha » $\mathrm{n}^{\circ}$ 9).

DORRONSORO Gilles, La révolution afghane. Des communistes aux Taleban, Paris, Karthala, 2000.

FANDY Mamoun, «Cyber Resistance: Saudi Opposition between Globalization and Localization », Comparative Studies in Society and History, 41, 1999, pp. 124-147.

FEILLARD Andrée, Islam et armée dans l'Indonésie contemporaine, Paris, L'Harmattan, 1995.

FEILlARD Andrée, éd., , L'islam en Asie, du Caucase à la Chine, Paris, La Documentation Française, 2001 (préface de Marc Gaborieau).

FRÉGOSI Franck, La formation des cadres religieux en France, Paris, L'Harmattan, 1998.

GABORIEAU Marc, «Les oulémas/soufis dans l'Inde moghole : anthropologie historique de religieux musulmans », Annales ESC, 1989, nº 5, pp. 1185-1204.

GABORIEAU Marc, «Les protestations d'un soufi indien contemporain contre trois interprétations récentes de Shaikh Ahamad Sirhindi », in Marc GABORIEAU, Alexandre POPOVIC, Thierry ZARCONE, éds., Naqshbandis. Cheminements et situation actuelle d'un ordre mystique musulman, Istanbul, Éditions Isis, 1990, pp. 237-267.

GABORIEAU Marc, «The Transmission of Islamic Reformist Teachings to Rural South Asia. The Lessons of a Case Study ", in Hassan ELBOUDRARI, éd., Modes de transmission de la culture religieuse en Islam, Le Caire, Institut Français d'Archéologie Orientale, 1993, pp. 119-157.

GABORIEAU Marc, « Renouveau de l'islam ou stratégie politique occulte ? La Tablîghî Jamâ'at dans le sous-continent indien et dans le monde », in Catherine CLÉMENTIN-OJHA, éd., Renouveaux religieux en Asie, Paris, École française d'Extrême-Orient, 1997, pp. 211-229.

GABORIEAU Marc, « Islam et politique », in Christophe JAFFRELOT, éd., Le Pakistan, Paris, Fayard, 2000, pp. 399-422.

GAFFNEY Patrick, The Prophet's Pulpit: Islamic Preaching in Contemporary Egypt, Berkeley, University of California Press, 1994.

GARDET Louis, L'islam. Religion et communauté, $2^{\text {ème }}$ éd., Paris, Desclée de Brouwer, 2002 (1 ère éd., 1967).

GILSENAN Michael, «Some factors in the decline of Sufi orders in Modern Egypt», The Muslim World, 57, 1967, pp. 11-18.

GRANDIN Nicole, GABORIEAU Marc, éds., Madrasa. La transmission du savoir dans le monde musulman, Paris, Éditions Arguments, 1997.

GRARE Frédéric, Political Islam in the Indian Subcontinent. The Jamaat-i-Islami, Delhi, Manohar, 2001.

GRIL Denis, "Spiritualités", in Jean-Claude GARCIN et alii, États, sociétés et cultures du monde musulman médiéval, $X^{e}-X V^{e}$ siècle, Tome 2, Sociétés et cultures, 2000, pp. 421-452.

HARDY Peter, The Muslims of British India, Cambridge, Cambridge University Press, 1972 (réimpression, Delhi, Cambridge in India, 1998).

HARDY Peter, "The Authority of Muslim Kings in Mediaeval India », in Marc GABORIEAU, éd., Islam et société en Asie du Sud, Paris, Éditions de l'EHESS, 1986, pp. $37-55$ (coll. «Purushârtha» $\mathrm{n}^{\circ}$ 9).

HAMÈS Constant, «L'Europe occidentale contemporaine », in Alexandre POPOVIC, Gilles VEINSTEIN, éds., Les voies d'Allah. Les ordres mystiques dans le monde musulman des origines à aujourd'hui, Paris, Fayard, 1996.

HAMMOUDI Abdellah, Master and Disciple, Chicago, University of Chicago Press, 1997.

HERVIEU-LÉGER Danièle, «Quelques paradoxes de la modernité religieuse », Futuribles, n 260, 2001, pp. 99-109. 
KEDDIE Nikkie, éd., Scholars, Saints and Sufis: Muslim Religious Institutions in the Middle East Since 1500, Berkeley, University of California Press, 1972.

LAPIDUS Ira, "The Separation of State and Religion in the Development of Early Islamic Society », International Journal of Middle East Studies, VI, 1975, pp. 363-385.

MAYEUR-JAOUEN Catherine, éd., Saints et héros du Moyen-Orient contemporain, Paris, Maisonneuve et Larose, 2002.

MERVIN Sabrina, Histoire de l'islam. Doctrines et fondements, Paris, Flammarion, 2000, (coll. « Champs-Université »).

METCALF Barbara Daly, Islamic Revival in British India, Princeton, Princeton University Press, 1982.

NASR Seyyed Vali Reza, Mawdudi \& the Making of Islamic Revivalism, New York, Oxford University Press, 1996.

POPOVIC Alexandre, VEINSTEIN Gilles, éds., Les voies d'Allah. Les ordres mystiques dans le monde musulman des origines à aujourd'hui, Paris, Fayard, 1996.

RICHARD Yann, "Le rôle du clergé : tendances contradictoires du chi'isme iranien contemporain », Archives de sciences sociales des religions, $\mathrm{n}^{\circ}$ 55.1, 1983, pp. 5-27.

ROY Olivier, "L'islam, facteur de la politique étrangère (Asie centrale et monde arabo-persan) », in Christophe JAFFRELOT, éd., Le Pakistan, Paris, Fayard, 2000, pp. 235-257.

ROY Olivier, L'islam mondialisé, Paris, Seuil, 2002.

SANYAL Usha, Devotional Islam \& Politics in British India. Ahmad Riza Khan Barelwi and his Movement, 1870-1920, Delhi, Oxford University Press, 1996.

SADOWSKY Yahia, « The New Orientalism and the Democracy Debate », in Joel BENIN, Joe STORK, éds., Political Islam, Essays from Middle East Report, Berkeley, University of California Press, 1997, pp. 33-50.

TOUATI Houari, Entre Dieu et les hommes. Lettrés, saints et sorciers au Maghreb (17e siècle), Paris, Éditions de l'EHESS, 1994.

ZAMAN Muhammad Qasim, Religion and Politics under the Early 'Abbâsids. The Emergence of the Proto-Sunnî Elite, Leyde, E. J. Brill, 1997.

ZAMAN Muhammad Qasim, The Ulama in Contemporary Islam, Custodians of Change, Princeton, Princeton University Press, 2002.

ZEGHAL Malika, Gardiens de l'islam, Paris, Presses de Sciences-Po, 1996.

ZEGHAL Malika, " La guerre des fatwa-s. Cheikhs Taantawî et Gad al-Haqq à l'épreuve du pouvoir », Cahiers de l'Orient, 45, janvier-mars 1997, pp. 81-95.

ZEGHAL Malika, «État et marché des biens religieux. Les Voies égyptienne et tunisienne », Critique internationale, $\mathrm{n}^{\circ}$ 5, octobre 1999a, pp. 75-95.

ZEGHAL Malika, « Religion and Politics in Egypt: The Islamists, the Ulema and the State (1852-1994) », International Journal of Middle East Studies, volume 31,3, août 1999b, pp. 371-399. 
